\title{
Ginsenoside compound $K$ promotes $\beta$-amyloid peptide clearance in primary astrocytes via autophagy enhancement
}

\author{
JINHUI GUO ${ }^{1}$, LI CHANG ${ }^{2}$, XIN ZHANG $^{1}$, SUJUAN PEI $^{1}$, MEISHUANG YU $^{1}$ and JIANLIAN GAO ${ }^{1}$ \\ Departments of ${ }^{1}$ Pharmaceutics and ${ }^{2}$ Neurology, The First Affiliated Hospital of Xinxiang Medical University, \\ Weihui, Henan 453100, P.R. China
}

Received February 13, 2014; Accepted July 25, 2014

DOI: $10.3892 /$ etm.2014.1885

\begin{abstract}
The aim of the present study was to investigate the effect of ginsenoside compound $K$ on $\beta$-amyloid $(A \beta)$ peptide clearance in primary astrocytes. $A \beta$ degradation in primary astrocytes was determined using an intracellular $A \beta$ clearance assay. Aggregated LC3 in astrocyte cells, which is a marker for the level of autophagy, was detected using laser scanning confocal microscope. The effect of compound $\mathrm{K}$ on the mammalian target of rapamycin (mTOR)/autophagy pathway was determined using western blot analysis, and an enzyme-linked immunosorbent assay was used for $\mathrm{A} \beta$ detection. The results demonstrated that compound $\mathrm{K}$ promoted the clearance of $\mathrm{A} \beta$ and enhanced autophagy in primary astrocytes. In addition, it was found that phosphorylation of mTOR was inhibited by compound $\mathrm{K}$, which may have contributed to the enhanced autophagy. In conclusion, compound $\mathrm{K}$ promotes $\mathrm{A} \beta$ clearance by enhancing autophagy via the mTOR signaling pathway in primary astrocytes.
\end{abstract}

\section{Introduction}

Alzheimer's disease (AD) is a progressive neurodegenerative disease of the central nervous system, with memory loss as the primary clinical manifestation. As a result of the aging population, the number of patients with $\mathrm{AD}$ is predicted to be $>100$ million in 2050 worldwide (1). However, at present, there are no effective drugs that delay the progression of AD.

Previous studies have demonstrated that $\beta$-amyloid $(\mathrm{A} \beta)$ peptides have an important role in AD. Aggregation of $A \beta$ may cause neurofibrillary tangles, inflammation and neuronal loss, resulting in the development of $\mathrm{AD}(2)$. It is thought that reduced generation or accelerated clearance of $A \beta$ may delay the pathological progression of $\mathrm{AD}(3,4)$. Autophagy is considered to be an

Correspondence to: Dr Jinhui Guo, Department of Pharmaceutics, The First Affiliated Hospital of Xinxiang Medical University, No. 88 Jiankang Road, Weihui, Henan 453100, P.R. China

E-mail: jinhuiguocn@126.com

Key words: Alzheimer's disease, compound K, $\beta$-amyloid peptides, autophagy important regulatory factor in A $\beta$ clearance. During autophagy, $\mathrm{A} \beta$ is taken up by autophagic vacuoles, which then fuse to the lysosome and are degraded $(5,6)$. Therefore, autophagy enhances $A \beta$ clearance. It has been previously demonstrated that mammalian target of rapamycin (mTOR) kinase is an important energy sensitivity factor. mTOR is inhibited when there is lack of energy, and inhibition of mTOR results in the activation of autophagy to produce additional energy $(7,8)$. Therefore, inhibition of mTOR may enhance autophagy, and as a result increase the clearance of intracellular $A \beta$, thereby delaying the pathological progression of AD (9).

Panax ginseng is a traditional Chinese medicine, which has been used as a medicine for thousands of years. Studies have shown that ginseng has a number of biological activities, including as an antioxidant, anti-aging agent, inhibitor of cell apoptosis and cognition enhancer $(10,11)$. Ginsenosides are active compounds extracted from ginseng, and it has been demonstrated that ginsenoside $\mathrm{Rg} 1$ is able to improve memory and has anti-dementia effects (12). Ginsenoside compound $\mathrm{K}$ is a metabolite of panaxadiol (a saponin) that is generated by the metabolic action of intestinal flora in humans. It is considered that numerous ginsenosides are metabolized into compound $\mathrm{K}$ prior to becoming active in vivo. Compound $\mathrm{K}$ is hypothesized to be the active compound in vivo, while ginsenosides may be prodrugs that have no effect (13). Therefore, the pharmacological mechanisms of compound $\mathrm{K}$ require further investigation. In present study, the $\mathrm{A} \beta$-scavenging effect of compound $\mathrm{K}$ and the underlying mechanisms were investigated in primary astrocytes.

\section{Materials and methods}

Primary culturing of mouse astrocytes. C57 mice were purchased from the Experimental Animal Center of Xinxiang Medical University (Xinxiang, China) and at 18 days of pregnancy were anaesthetized using pentobarbital and then paunched. The fetal mice were removed and placed into pre-cooling D-Hank's buffer. The brains of the fetal mice were removed and the meninges were discarded. The cerebral cortex was separated from the brain tissues and then digested for $15 \mathrm{~min}$ at $37^{\circ} \mathrm{C}$ in $8 \mathrm{ml} 0.125 \%$ trypsin $(2.5 \%$ trypsin diluted 20-fold with D-Hank's and DNase, at a final concentration of $0.2 \mathrm{mg} / \mathrm{ml}$ ). Digestion was terminated by the addition of $8 \mathrm{ml}$ Dulbecco's modified Eagle's medium (Gibco, Grand Island, 
NY, USA) containing $10 \%$ horse serum (Gibco) for $5 \mathrm{~min}$. The upper suspension was transferred to a $50-\mathrm{ml}$ tube and an additional $8 \mathrm{ml}$ complete growth medium was added to the deposit and the previous step was repeated. The cells in the suspension were placed at a density of $2 \times 10^{5} \mathrm{cell} / \mathrm{cm}^{2}$ in a $75-\mathrm{cm}^{2}$ flask that was pre-coated with poly-D-lysine. The cells were incubated at $37^{\circ} \mathrm{C}$ and $5 \% \mathrm{CO}_{2}$ for 9 days, and the medium was replaced every three days. The flask was then placed on a ZHWY-110X shaker table (Zhicheng Co., Shanghai, China) at $200 \mathrm{rpm}$ at $37^{\circ} \mathrm{C}$ overnight. On the second day, the flask was rinsed twice with D-Hank's at $37^{\circ} \mathrm{C}$, and the remaining adherent cells were collected with trypsin and subcultured. Following attachment, the cells were treated with Ara-C for $96 \mathrm{~h}$ (medium renewal once) at a final concentration of $10 \mu \mathrm{M}$, and the cells obtained were astrocytes. This study was performed in strict accordance with the recommendations in the Guide for the Care and Use of Laboratory Animals of the National Institutes of Health (8th edition, Bethesda, MD, USA, 2010). The animal use protocol was reviewed and approved by the Institutional Animal Care and Use Committee of the First Affiliated Hospital of Xinxiang Medical University (Weihui,, China).

Detection of aggregated LC3. A viral expression vector containing a red fluorescent protein-LC3 construct (Jikai Biotechnology Co. Ltd., Shanghai, China) was transfected into the primary astrocytes. Transfection was performed using a Sunma-sofast gene transfection kit according to the manufacturer's instructions (Sunma Biotechnology Co., Ltd., Xiamen, China). LC3 proteins were then confirmed to be stably expressed in the cells. After two days, the cells were treated with $50 \mu \mathrm{M}$ compound $\mathrm{K}$ (Fangcheng Biotechnology Co. Ltd., Beijing, China) for $2 \mathrm{~h}$, and the aggregation state of the LC3 proteins was detected by confocal laser scanning microscopy (TCS-SP5, Leica, Bensheim, Germany).

Western blot analysis. Cells treated with different concentration of compound $\mathrm{K}$ were treated with radio-immunoprecipitation assay lysis buffer at $75^{\circ} \mathrm{C}$ for $10 \mathrm{~min}$. The lysates were incubated at $99^{\circ} \mathrm{C}$ for $15 \mathrm{~min}$, and then centrifuged at $10,000 \mathrm{x} \mathrm{g}$ for $5 \mathrm{~min}$ at $4^{\circ} \mathrm{C}$. The supernatant was separated using SDS-PAGE and then transferred to a polyvinylidene difluoride membrane under semi-dry electrotransfer conditions. The membrane was incubated in blocking buffer [1X Tris-buffered saline (TBS), $0.5 \%$ Tween 20 and $5 \%$ skimmed milk powder] for $30 \mathrm{~min}$, followed by incubation with the primary antibody (targeting P70S6K, P70S6K-P, ULK1, ULK1-P, mTOR, mTOR-P, P62 or GAPDH; Cell Signaling Technology Inc., Beverly, MA, USA) at $4^{\circ} \mathrm{C}$ overnight. The membranes were then rinsed 3 times with TBS and Tween 20 (TBST) for $15 \mathrm{~min}$, and then incubated with the corresponding monoclonal, anti-rabbit secondary antibody (Santa-Cruz Biotechnology Inc., Santa Cruz, CA, USA) for $2 \mathrm{~h}$, prior to being rinsed again with TBST 3 times. The membrane was then incubated with SuperSignal West Dura Chemiluminescent Substrate (Pierce, Thermo Scientific, Rockford, IL, USA) for $5 \mathrm{~min}$ and images were captured by $\mathrm{X}$-ray film exposure.

$A \beta$ clearance assay. The $\mathrm{A} \beta$ clearance assay in primary astrocytes was performed as previously described by Cramer et al (14). Briefly, the cells were incubated with different concentrations of compound $\mathrm{K}(0,1,10,20$ and $50 \mu \mathrm{M}$ ) for $18 \mathrm{~h}$, meanwhile, chloroquine (an inhibitor of autophagy) was used as a control and then exogenous $A \beta$ (Invitrogen Life Technologies, Carlsbad, CA, USA) was added to a final concentration of $2 \mu \mathrm{g} / \mathrm{ml}$. The cells were then incubated for a further $3 \mathrm{~h}$. The cells were washed 3 times with phosphate-buffered saline, and then treated with lysis buffer (50 $\mathrm{mM}$ Tris and $1 \%$ SDS) at $37^{\circ} \mathrm{C}$ for $15 \mathrm{~min}$. The lysates were centrifuged at $12,000 \mathrm{x}$ g for $15 \mathrm{~min}$, and the supernatant was collected. $A \beta$ was then quantified using an enzyme-linked immunosorbent assay (ELISA kit for $\mathrm{A} \beta$ detection).

ELISA. An ELISA for A $\beta$ detection was conducted in accordance with the manufacturer's instructions (Invitrogen Life Technologies). The diluted samples were incubated with $\mathrm{A} \beta$ antibody in a 96-well plate that was pre-coated with $\mathrm{A} \beta$ antibody. After $3 \mathrm{~h}$, the plate was rinsed with cleaning solution (Biyuntian Co., Shanghai, China) four times, and then incubated with the secondary antibody for $30 \mathrm{~min}$ and rinsed five times. The chromogenic substrate was then added and the plates were incubated for a further $30 \mathrm{~min}$. Finally the reaction was terminated using stop solution. The intensity of color developed was measured using microplate reader (Bio-Rad 680, Bio-Rad, Hercules, CA, USA) at $570 \mathrm{~nm}$. In order to eliminate the interference of the cell density, the cells were lysed (50 mm Tris-HCl, $0.15 \mathrm{M}$ sodium chloride, 1\% P40 and $0.1 \%$ SDS) and the protein content was measured using the bicinchoninic acid assay method. The measured density was adjusted according to the total protein content.

Statistical analysis. The data are expressed as the mean \pm standard deviation and were analyzed using SPSS software, version 16.0 (SPSS,Inc., Chicago, IL, USA). One-way analysis of variance was used to compare the scores of different groups. $\mathrm{P}<0.05$ was considered to indicate a statistically significant difference.

\section{Results}

Compound $K$ promotes clearance of $A \beta$ in primary astrocytes. The levels of $\mathrm{A} \beta$ in astrocytes treated with compound $\mathrm{K}$ were significantly lower compared with those in untreated astrocytes. The differences were significantly different for the 10,20 and $50 \mu \mathrm{M}$ concentrations of compound $\mathrm{K}(\mathrm{P}<0.001$; Fig. 1). These results indicate that compound $K$ enhances $A \beta$ clearance in primary astrocytes. In order to investigate the association between compound $\mathrm{K}$ and autophagy, chloroquine, an inhibitor of autophagy, was used as a control. The results demonstrated that chloroquine markedly attenuated the effect of compound $\mathrm{K}$ on the enhancement of $\mathrm{A} \beta$ clearance. This indicates that compound $\mathrm{K}$ promotes $\mathrm{A} \beta$ clearance through the enhancement of autophagy in primary astrocytes.

Compound $K$ enhances autophagy in primary astrocytes. Autophagy has an important role in $A \beta$ clearance. In order to further clarify the association between autophagy and $A \beta$ clearance, the effect of compound $K$ on autophagy was investigated. LC3 is an important marker of autophagosome formation. When autophagy is enhanced, LC3 proteins that are dispersed throughout the cell gather to the membrane 
A

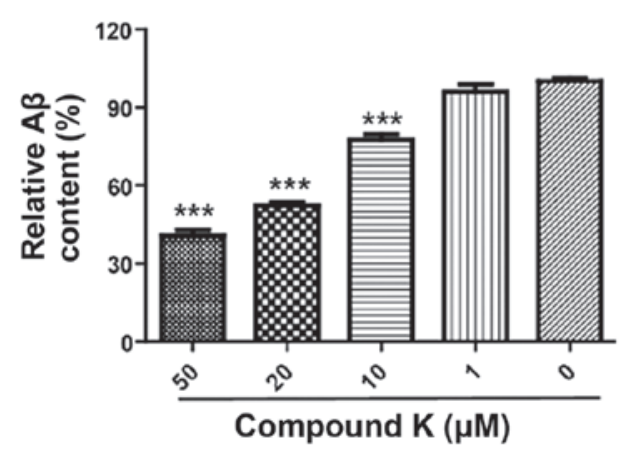

B

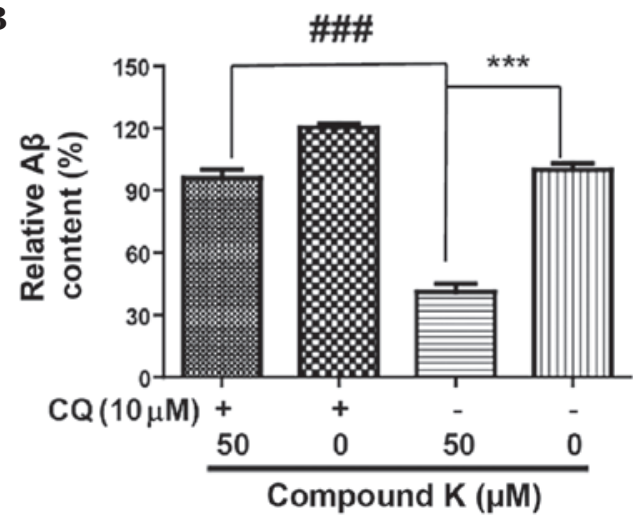

Figure 1. Compound K promotes clearance of A $\beta$ in primary astrocytes. (A) Primary astrocytes were treated with different concentrations of compound $\mathrm{K}$ (50, 20,10, 1 and $0 \mu \mathrm{M}$ ) for $18 \mathrm{~h}$. Exogenous $\mathrm{A} \beta$ was then added and the cells were incubated for a further $3 \mathrm{~h}$. Then the cells were lysed and the content of $\mathrm{A} \beta$ was measured by ELISA. (B) Primary astrocytes were treated with $50 \mu \mathrm{M}$ compound $\mathrm{K}$ or $10 \mu \mathrm{M}$ chloroquine or a combination of the two for $18 \mathrm{~h}$. Exogenous $\mathrm{A} \beta$ was added and the cells were incubated for $3 \mathrm{~h}$. The cells were then lysed and the A $\beta$ content was measured by ELISA $(\mathrm{n}=3) .{ }^{* * *} \mathrm{P}<0.001 \mathrm{compared}$ with the $0 \mu \mathrm{M}$ compound $\mathrm{K}$ group, ${ }^{\# \# \#} \mathrm{P}<0.001$.

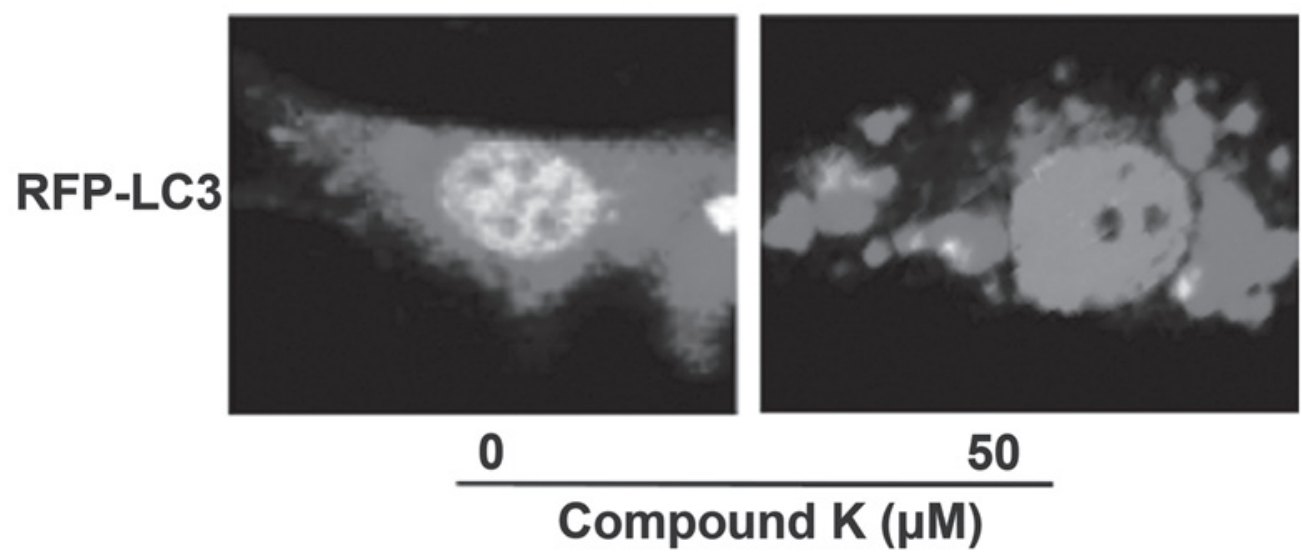

Figure 2. Compound K enhances autophagy in primary astrocytes. Primary astrocytes were transfected with LC3 and and stably expressed the protein. After 2 days, cells were incubated with $50 \mu \mathrm{M}$ compound $\mathrm{K}$ for $2 \mathrm{~h}$. The aggregation state of LC 3 was then detected by laser scanning confocal microscopy.
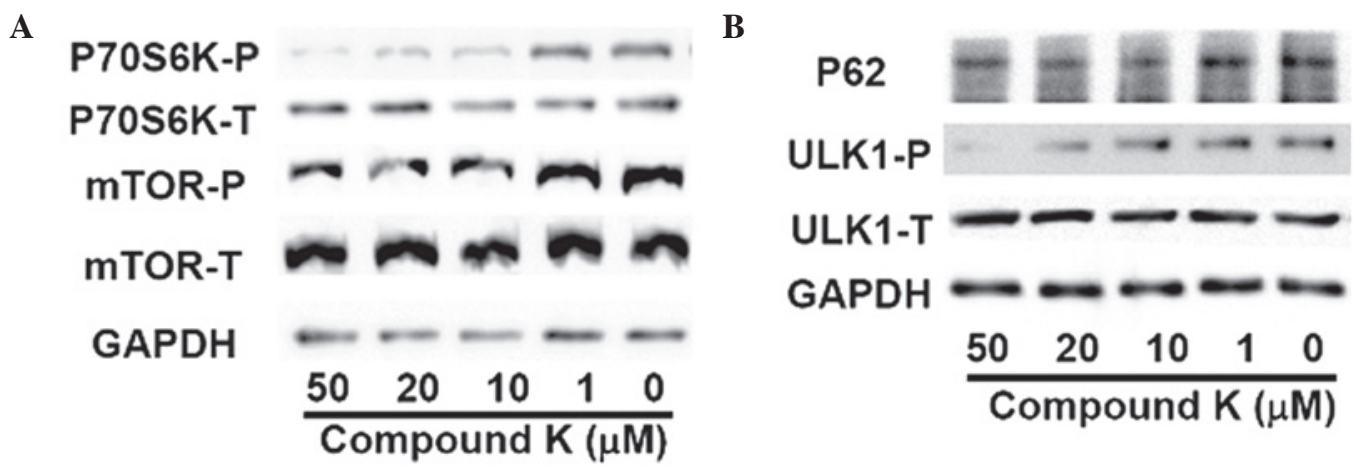

Figure 3. Compound K enhances autophagy by inhibiting the activity of mTOR. (A) Primary astrocytes were treated with different concentrations of compound $\mathrm{K}(50,20,10,1$ or $0 \mu \mathrm{M})$ for $24 \mathrm{~h}$. The total proteins were collected and the phosphorylation levels of P70S6K and mTOR were detected by western blot analysis. (B) Primary astrocytes were treated with different concentrations of compound $\mathrm{K}(50,20,10,1$ or $0 \mu \mathrm{M})$ for $24 \mathrm{~h}$. The total proteins were collected and the phosphorylation of ULK1 and the levels of P62 were detected using western blot analysis. mTOR, mammalian target of rapamycin.

of autophagosome, which can then be detected using laser scanning confocal microscopy. Following transfection with LC3, the primary astrocytes were found to stably express LC3 protein. It was observed that LC3 proteins aggregated following incubation with $50 \mu \mathrm{M}$ compound $\mathrm{K}$ for $2 \mathrm{~h}$. This indicates that compound $\mathrm{K}$ enhances autophagy in astrocytes, which may contribute to increased clearance of $A \beta$ (Fig. 2).

Compound $K$ enhances autophagy by inhibiting the mTOR signal pathway. As an important factor of energy sensitivity, 
mTOR kinase is inhibited when there is an energy shortage, leading to an enhancement of autophagy. Therefore, the phosphorylation levels of mTOR and P70S6K, the substrate of mTOR, in primary astrocytes treated with compound $\mathrm{K}$ were investigated by western blot analysis. As shown in Fig. 3A, the phosphorylation levels of mTOR and P70S6K were reduced by compound $\mathrm{K}$, indicating that compound $\mathrm{K}$ enhances autophagy by inhibiting the activity of mTOR. To further investigate this, the phosphorylation of ULK1, the major initiator protein of autophagy, and the levels of P62, the marker of the autophagy, were then investigated (15). As shown in Fig. 3B, compound K significantly reduced the expression level of P62 and the phosphorylation of ULK1, indicating that compound K activates and enhances autophagy in astrocytes.

These results suggest that compound $\mathrm{K}$ may have an inhibitory effect on the phosphorylation of mTOR and subsequently enhance autophagy, which may contribute to increased scavenging of $A \beta$ in primary astrocytes.

\section{Discussion}

$\mathrm{A} \beta$ is considered to be one of the key proteins in $\mathrm{AD}$ progression. In vivo, $\mathrm{A} \beta$ is in a dynamic equilibrium between generation and scavenging, and excess generation or weak scavenging of $A \beta$ is the main pathogeny of AD. Drugs that reduce $A \beta$ production or increase the clearance of $A \beta$ may slow $A D$ progression. Autophagy is the most important pathway for the clearance of abnormal molecules, cell subunits and abnormal aggregates of proteins. Autophagy levels have been found to be significantly decreased in various neurodegenerative diseases, including AD and Parkinson's disease $(16,17)$. Therefore, enhanced autophagy may increase the clearance of $A \beta$, which may delay the pathological process of $\mathrm{AD}$. In a previous study it was reported that resveratrol and its derivatives from grape seeds enhance autophagy through AMPK activation and subsequently the inhibition of mTOR, resulting in the scavenging of $A \beta$, which may have a role in the treatment of AD (18). In addition, latrepirdine, a type of antihistamine that is already on the market, has been demonstrated to increase the clearance of $A \beta$ by enhancing autophagy, and has a role in the treatment of $A D$ (19). Therefore, compounds that are able to enhance the scavenging of $A \beta$ by promoting autophagy may be effective in AD therapy, and the screening of this type of compound is valuable for the treatment of AD.

Previous studies have shown that Panax ginseng has a number of biological activities, including the ability to improve memory. Compound $\mathrm{K}$ is the metabolite of numerous ginsenosides in the intestine, and is the main active entity of Panax ginseng in vivo. It has previously been shown that ginsenosides are able to upregulate the expression of brain-derived neurotrophic factor and inhibit the phosphorylation of Tau, which results in slowing of the formation of neurofibrillary tangles and should improve learning ability and memory. In addition, it has been shown to have an anti-AD role in a rat model of AD (20).

The effect of compound $K$ on $A \beta$ scavenging has yet to be fully elucidated. In the present study, the effect of compound $\mathrm{K}$ on $\mathrm{A} \beta$ scavenging and the mechanisms involved was investigated using the $A \beta$ clearance assay in vitro. The results demonstrated that compound $\mathrm{K}$ significantly enhanced the clearance of $A \beta$ in primary astrocytes. Furthermore, it was shown that the phosphorylation of mTOR was inhibited by compound $\mathrm{K}$, which may contribute to enhanced autophagy, and thereby increase the scavenging of $\mathrm{A} \beta$. In conclusion, these results indicate that compound $\mathrm{K}$ may increase the clearance of $A \beta$ from astrocytes and then slow the pathological progression of AD. This study provides an important basis for the use of Panax ginseng as a traditional Chinese medicine for improving memory, and provides a novel interpretation of the mechanism of compound $\mathrm{K}$ in $\mathrm{AD}$ therapy.

\section{References}

1. Brookmeyer R, Johnson E, Ziegler-Graham K and Arrighi HM: Forecasting the global burden of Alzheimer's disease. Alzheimers Dement 3: 186-191, 2007.

2. Younkin SG: The role of A beta 42 in Alzheimer's disease. J Physiol Paris 92: 289-292, 1998.

3. Ohno M, Sametsky EA, Younkin LH, et al: BACE1 deficiency rescues memory deficits and cholinergic dysfunction in a mouse model of Alzheimer's disease. Neuron 41: 27-33, 2004.

4. Jarvis CI, Goncalves MB, Clarke E, et al: Retinoic acid receptor- $\alpha$ signalling antagonizes both intracellular and extracellular amyloid- $\beta$ production and prevents neuronal cell death caused by amyloid- $\beta$. Eur J Neurosci 32: 1246-1255, 2010.

5. Bachmeier C, Beaulieu-Abdelahad D, Mullan M and Paris D: Selective dihydropyiridine compounds facilitate the clearance of $\beta$-amyloid across the blood-brain barrier. Eur J Pharmacol 659: 124-129, 2011.

6. Kennelly SP, Abdullah L, Paris D, et al: Demonstration of safety in Alzheimer's patients for intervention with an anti-hypertensive drug Nilvadipine: results from a 6-week open label study. Int J Geriatr Psychiatry 26: 1038-1045, 2011.

7. Klionsky DJ and Emr SD: Autophagy as a regulated pathway of cellular degradation. Science 290: 1717-1721, 2000.

8. Zhu Z, Yan J, Jiang W, et al: Arctigenin effectively ameliorates memory impairment in Alzheimer's disease model mice targeting both $\beta$-amyloid production and clearance. J Neurosci 33: 13138-13149, 2013.

9. Spilman P, Podlutskaya N, Hart MJ, et al: Inhibition of mTOR by rapamycin abolishes cognitive deficits and reduces amyloid-beta levels in a mouse model of Alzheimer's disease. PLoS One 5: e9979, 2010.

10. Cho CW, Kim YC, Kang JH, et al: Characteristic study on the chemical components of Korean curved ginseng products. J Ginseng Res 37: 349-354, 2013.

11. Seo SK, Hong Y, Yun BH, et al: Antioxidative effects of Korean red ginseng in postmenopausal women: A double-blind randomized controlled trial. J Ethnopharmacol 154: 753-757, 2014.

12. Zhang X, Wang J, Xing Y, et al: Effects of ginsenoside Rg1 or $17 \beta$-estradiol on a cognitively impaired, ovariectomized rat model of Alzheimer's disease. Neuroscience 220: 191-200, 2012.

13. Wakabayashi C, Murakami K, Hasegawa H, Murata J and Saiki I: An intestinal bacterial metabolite of ginseng protopanaxadiol saponins has the ability to induce apoptosis in tumor cells. Biochem Biophys Res Commun 246: 725-730, 1998.

14. Cramer PE, Cirrito JR, Wesson DW, et al: ApoE-directed therapeutics rapidly clear beta-amyloid and reverse deficits in AD mouse models. Science 335: 1503-1506, 2012.

15. Mizushima N: The role of the Atg1/ULK1 complex in autophagy regulation. Curr Opin Cell Biol 22: 132-139, 2010.

16. Hara T, Nakamura K, Matsui M, et al: Suppression of basal autophagy in neural cells causes neurodegenerative disease in mice. Nature 441: 885-889, 2006.

17. Nixon RA: The role of autophagy in neurodegenerative disease. Nat Med 19: 983-997, 2013.

18. Vingtdeux V, Chandakkar P, Zhao H, et al: Novel synthetic small-molecule activators of AMPK as enhancers of autophagy and amyloid- $\beta$ peptide degradation. FASEB J 25: 219-231, 2011.

19. Steele JW and Gandy S: Latrepirdine (Dimebon ${ }^{\circledR}$ ), a potential Alzheimer therapeutic, regulates autophagy and neuropathology in an Alzheimer mouse model. Autophagy 9: 617-618, 2013.

20. Gao C, Liu Y, Jiang Y, Ding J and Li L: Geniposide ameliorates learning memory deficits, reduces tau phosphorylation and decreases apoptosis via GSK3 $\beta$ pathway in streptozotocininduced alzheimer rat model. Brain Pathol 24: 261-269, 2014. 